\title{
Stellar model choice and the MOPED fossil record
}

\author{
Benjamin Panter ${ }^{1}$ \\ ${ }^{1}$ SUPA $\dagger$, Institute for Astronomy, University of Edinburgh, Edinburgh EH9 3HJ, UK, \\ email: bdp@roe.ac.uk
}

\begin{abstract}
Recent spectral analysis of the SDSS DR3 has provided a wealth of information determined from the fossil record concerning star formation rates, stellar mass buildup, dust content and stellar mass functions. Essential to this work is an understanding of how model choice influences results gained from the analysis of galaxy spectra. We summarize the results of analyzing two plates of SDSS spectra with five different SSP models which appear in full in Panter et al. (2006) and discuss some residuals seen in a far larger sample of galaxies.
\end{abstract}

Keywords. methods: data analysis, methods: statistical, techniques: spectroscopic, surveys, galaxies: evolution, galaxies: formation, galaxies: fundamental parameters (classification, colors, luminosities, masses, radii, etc.), cosmology: observations

\section{Introduction}

The quality of spectra of the observed light of unresolved stellar populations has reached enough accuracy that it is possible to make detailed studies of the physical properties of the stellar populations in these galaxies. An excellent example of this new generation of data-sets is given by the Sloan Digital Sky Survey (Gunn et al. 1998; York et al. 2000; Strauss et al. 2002) at low redshift, not only by the size of the spectroscopic sample (about $10^{6}$ spectra) but by the quality and wavelength coverage of the spectra. Indeed, several attempts have been made previously to study in detail the physical properties of the SDSS galaxies either by using selected features in the spectra (Kauffmann et al. 2004; Brinchmann et al. 2004; Tremonti et al. 2004) or using the full spectrum (Panter, Heavens \& Jimenez 2003; Heavens et al. 2004; Panter, Heavens, \& Jimenez 2004; Cid Fernandes 2005; Mathis et al. 2006; Ocvirk et al. 2006). These studies have led to interesting conclusions about the physical properties of these galaxies. In particular analysis of the SDSS sample (Heavens et al. 2004) and other local galaxies (Thomas et al. 2005) show very clear evidence for 'downsizing' - the process by which star formation at low redshift takes place predominantly in low-mass galaxies, whereas more massive galaxies have the bulk of their star-formation activity at high redshift. All these methods share a dependence on stellar modelling codes. In this proceeding we summarize the effect of various choices on the Star Formation History ( $\mathrm{SFH}$ ) recovered from a reasonably sized $(\sim 850)$ set of galaxy spectra and discuss some residuals seen in a far larger sample of galaxies. This work is presented in full in Panter et al. (2006)

\section{Star Formation Fractions}

Fig. 1 shows the comparison between five different stellar population synthesis models for analysis of $\sim 850$ sloan galaxy spectra using the MOPED algorithm: the Jimenez et al. (2004) SPEED models, the Fioc \& Rocca-Volmerange (1997) PEGASE models,

$\dagger$ Scottish Universities Physics Alliance. 


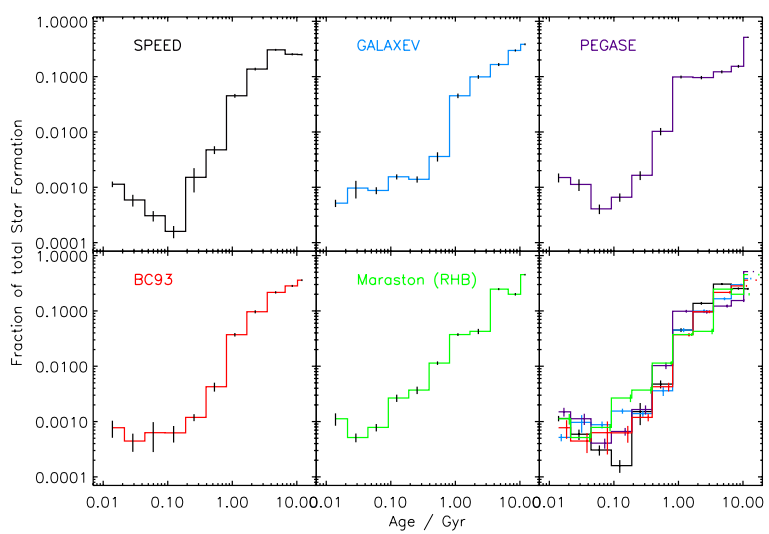

Figure 1. The systematic changes in recovered rest frame star formation fractions (not rates) for various different SSPs.

the Maraston (2005) RHB models; the Bruzual \& Charlot (1993) models and the more modern $3 \AA$ Bruzual \& Charlot (2003) GALAXEV models rebinned to $20 \AA$. The comparison is done at $20 \AA$ for the Salpeter IMF and for the one-parameter dust model. It is important to realise that this analysis cannot say which model set is 'right', only assist in understanding the differences between models. The overall shape of the recovered SFH is in reasonable agreement - although there are certainly discrepancies between the populations that are recovered. For the very oldest populations the different models agree very well. This is not entirely unexpected of course, as the stars which contribute to this area of the age-metallicity parameter space are well studied and dominate the emission at red wavelengths. The different models also predict roughly similar proportions of the very youngest populations, which rely on similar prescriptions for the evolution of blue massive stars and can be constrained through the emission at the bluest wavelengths. At intermediate ages the agreement is not so good: this is likely to be caused, at least in part, by the difficulty in recovering the fraction of intermediate-age stars in stellar populations with declining star formation histories (see discussion by Mathis et al. (2006)). This tends to produce an articifial step around 1 Gyr in the star formation history, except perhaps in the Maraston (2005) model. The different behavior of this model probably results from the different prescription for bright Asymptotic Giant Branch (AGB) stars. The contribution by these stars to the integrated light is still subject to controversy in current population synthesis models. Since our spectra are fitted in addition, any poorly fit component will be replaced by another.

Due to space restrictions, further discussion in Panter et al. (2006)

\section{Residuals of best fit models}

Due to the power of MOPED and the number of SSPs offered for fitting (11) excellent fits can be obtained if the models are accurate. By comparing the residuals of the best fitting spectrum to the raw data on a pixel by pixel basis and then averaging over many galaxies in the galaxy restframe it is possible to determine exactly which areas are not being accurately fitted for a given spectrum. By stacking the residuals of high signal to noise (Sloan SPECOBJALL.SCI_SN, science $S / N>20$ per flux measurement) galaxies it is possible to determine to a high degree of accuracy which wavelength ranges are failing in the models, and to what extent. If we assume that MOPED can, in most cases, obtain 


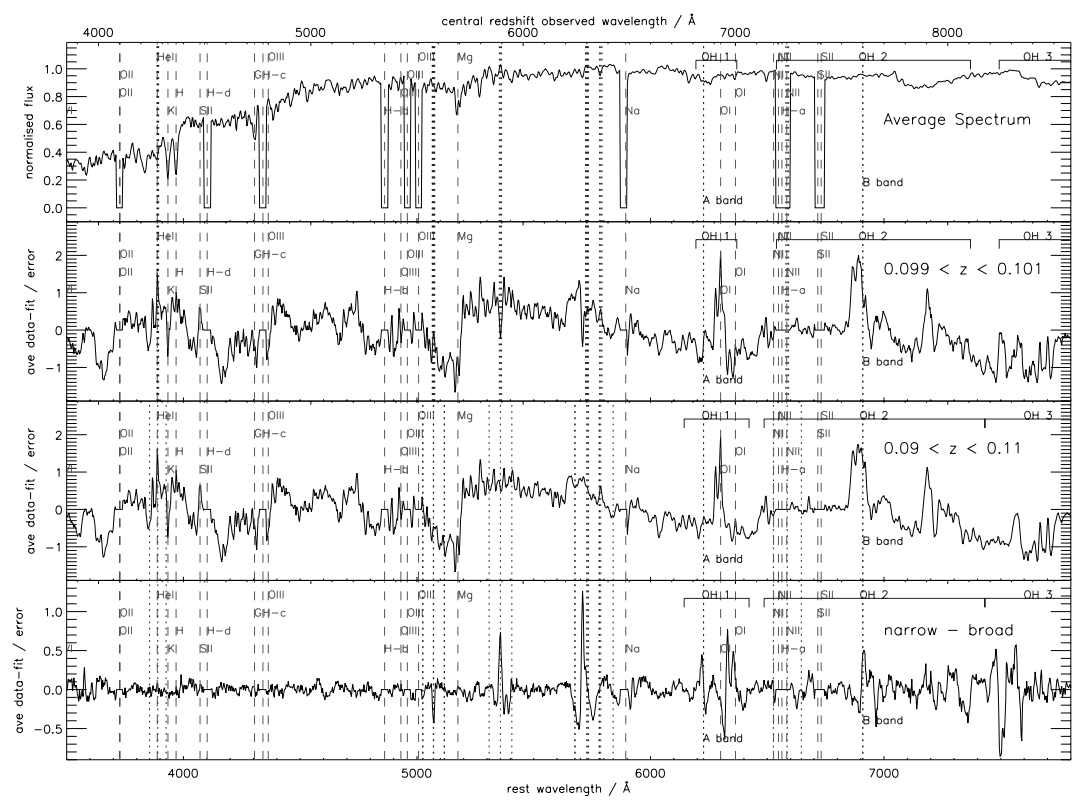

Figure 2. The first panel shows the average spectrum for the galaxies in with high $\mathrm{S} / \mathrm{N}$ and $z=0.1 \pm 0.01$. The second and third panels show the residual at each pixel divided by the error at that pixel averaged in the rest frame. The first plot shows the average for 747 high $\mathrm{S} / \mathrm{N}$ galaxies within the range $z=0.1 \pm 0.001$, the second for 7406 high $\mathrm{S} / \mathrm{N}$ galaxies within $z=0.1 \pm 0.01$. The third plot shows the first residual set minus the second, and clearly separates areas contaminated from skylines (which are broadened in the second plot by the redshift range). Known emission, absorption and skyline features are labelled in grey.

the best possible fit to a spectrum then the differences must be features not included in the models. These features could be things that the models are not designed to measure (instrumental effects, interstellar or intergalactic medium absorption, skyline contamination etc.) or alternatively features that are either misrepresented or not yet included in stellar modelling codes (e.g. alpha enhancement (Thomas, Maraston, \& Bender 2003), emission lines, helium production of $d Y / d Z \sim 1.5-2$ (Jimenez et al. 2003) and other spectral features). Fig 2 provides some insight into which features in the residuals can be identified. Since we subtract the modelled spectrum from the data, unfitted emission will have a positive effect on the residuals while unfitted absorption will be negative. Both obvious emission lines and filling of absorption features should be detectable. The spectra used for this analysis are those which have already had the strongest emission line regions removed, as detailed earlier - in this case the residual is simply zero.

The first panel of Fig. 2 shows the mean spectrum. To distinguish between galaxy features and skyline features we select two redshift ranges with the same central redshift, $z=0.1$. Since the averaging of residuals is carried out in the galaxy restframe, increasing the redshift spread will act to spread any skyline features. The third panel gives the residuals for galaxies within 0.001 of the central redshift while the second has a range of 0.01 . The fourth panel shows the second subtracted from the first. In this case, skyline/instrumental will create regions with large amplitude. The relevant skyline features (and their convolutions with the extremes of the particular redshift distribution) are shown and correspond exactly. It can be seen from the third panel that the majority 
of the spectral range is remarkably clear of skyline contamination - testament to the high quality of the Sloan spectroscopy.

Due to space restrictions, further discussion in Panter et al. (2006)

\section{Acknowledgements}

BP and SC thank the Alexander von Humboldt Foundation, the Federal Ministry of Education and Research, and the Programme for Investment in the Future (ZIP) of the German Government for funding through a Sofja Kovalevskaja award. The research of RJ is partially supported by NSF grants AST-0408698, PIRE-0507768 and NASA grant NNG05GG01G. BP wishes to thank Paul Hewett for considerable assistance identifying the source of various residuals in Fig. This analysis was performed using data from the Sloan Digital Sky Survey, please see http://www.sdss.org/collaboration/credits.html

\section{References}

Bruzual, A. G., \& Charlot, S., 1993, ApJ, 405, 538

Bruzual, G., \& Charlot, S., 2003, MNRAS, 344, 1000

Bell, E.F., McIntosh, D.H., Katz, N., \& Weinberg, M.D., ApJ, 2003, 585, 117-120

Bell, E. F., \& de Jong, R. S., 2001, ApJ, 550, 212

Brinchmann, J., Charlot, S., White, S. D. M., Tremonti, C., et al., 2004, MNRAS, 351, 1151

Cid Fernandes, R., Mateus, A., Sodré, L., Stasińska, G., \& Gomes, J. M. 2005, MRAS, 358, 363

Fioc M., \& Rocca-Volmerange, B., 1997, A\&A, 326, 950

Gunn et al. J. E., 1998, AJ, 116, 3040

Heavens, A., Panter, B., Jimenez, R., \& Dunlop, J. S., 2004, Nature

Jimenez, R., Flynn, C., MacDonald, J., \& Gibson, B. K., 2003, Science, 299, 1552

Jimenez, R., MacDonald, J., Dunlop, J. S., Padoan, P., \& Peacock, J. A., 2004, MNRAS, 349, 240

Kauffmann, G., White, S. D. M., Heckman, T. M., et al., 2004, astro-ph/0402030

Maraston, C., 2005, MNRAS, 362, 799

Mathis, H., Charlot, S., \& Brinchmann, J., 2006, MNRAS, 365, 385

Ocvirk, P., Pichon, C., Lançon, A., \& Thiébaut, E., 2006, MNRAS, 365, 46

Panter, B., Heavens, A. F., \& Jimenez, R., 2003, MNRAS, 343, 1145

Panter, B., Heavens, A. F., \& Jimenez, R., 2004,MNRAS, 355, 764

Panter, B., Thesis, 2005. http://hdl.handle.net/1842/774

Panter, B., Jimenez, R., Heavens, A. F., \& Charlot, S. 2006, ArXiv Astrophysics e-prints, arXiv:astro-ph/0608531

Salpeter, E. E., 1955, ApJ, 121, 161

Strauss et al. M. A., 2002, AJ, 124, 1810

Thomas, D., Maraston, C., \& Bender, R., 2003, MNRAS, 339, 897

Thomas, D., Maraston, C., Bender, R., \& Mendes de Oliveira, C. 2005, ApJ, 621, 673

Tremonti, C. A., Heckman, T. M., Kauffmann, G., et al. 2004, ApJ, 613, 898

York et al. D., 2000, AJ, 120, 1579

\section{Discussion}

OCVIRK: Did you try to interpret the residuals in the light of flux calibration issues in the SDSS?, i.e. comparing the residuals with the flux calibration error estimates obtained from comparisons of white dwarfs catalogs with models.

PANTER: I would caution that these areas should be removed by the smearing in the wide redshift range sample. Calibration issues should appear in the observing frame however, and I would be interested to see whether any links could be spotted. I have investigated the area around the dichroic carefully, but it is hard to attribute any specific features in the residuals to this wavelength range. 
PICKLES: After MOPED compression, how many parameters are used in the fit?, what is compression ratio?

PANTER: The number of parameters used in the fit is 23 , in this case 2 per population (of which there are 11) plus one dust parameter. The MOPED algorithm is quite general however and could equally be applied to any choice of parametrization. In terms of fitting, the algorithm produces a b-vector for each parameter, and the merit function for a given set of parameters consider the projections of the b-vector onto the data and models. In this way only 23 numbers, rather than 3000, are used for the fit, giving a compression ratio of $3000 / 23$.

Cid-Fernandes: It would be interesting to see your residual spectra split up into different galaxy types. When we do that, grouping, say star forming or elliptical galaxies, interesting differences appear, some of these residuals are well understood, like $\alpha$ residuals, whereas others, like a broad residual around $\mathrm{H} \beta$, maybe more related to get uncharted model deficiencies.

PANTER: I agree that this would be a very interesting study. As yet, the only analysis which we have performed is to split the residuals by galaxy type and look for symptoms of $\alpha$ enhancement, which we see cleary, as predicted by Thomas et al. I look forward to this analysis!.

Rose: The MOPED analysis uses 11 age populations that are binned logartithmically in time, which matches the fact that the galaxy spectrum evolves non-linearly with time. Is it possible that by establishing your time bins in this way you are biasing yourself forwards a solution a 4 Gyr old (in light weighted mean age) galaxy as a combination of a 1 Gyr and 10 Gyr composite?. In other words, are you maybe biasing yourself towards solutions with young populations?

PANTER: The only problem I can see is that if the bin is too wide then the composite spectrum assumed for that spectrum will not properly reflective of the extremes of that bin. However we choose bin boundaries in log time specifically to minimise this problem, or at least to ensure that it has equal impact on every bin. Of course, if by coincidence a 4 Gyr galaxy spectrum is better fit by the composite of two populations than the one of the bin what properly contains it the $\mathrm{n}$ the method will pick the incorrect solution. We have performed many tests to try to see if bias is introduced by MOPED analysis (Panter 2003) but see not such effect. The results of the stellar population challenge at this conference may suggest an area of improvement however. 